\title{
tic\&société
}

Vol. $4, n^{\circ} 1 \mid 2010$

Interactivité et lien social

\section{Langages structurés et lien social}

\section{Alain Vaucelle et Henri Hudrisier}

\section{OpenEdition}

\section{Journals}

Édition électronique

URL : http://journals.openedition.org/ticetsociete/790

DOI : 10.4000/ticetsociete. 790

Éditeur

Association ARTIC

Référence électronique

Alain Vaucelle et Henri Hudrisier, «Langages structurés et lien social », tic\&société [En ligne], Vol. 4, n 1 | 2010, mis en ligne le 17 mai 2010, consulté le 01 mai 2019. URL : http://journals.openedition.org/ ticetsociete/790 ; DOI : 10.4000/ticetsociete.790 
tic\&société - 4 (1), 2010

\title{
Langages structurés et lien social
}

\author{
Alain Vaucelle \\ Institut Télécom, Télécom SudParis \\ Dpt. Artémis, Université Paris 8, laboratoire Paragraphe. \\ 11 impasse Jacques Auguste, \\ F - 93400 Saint-Ouen \\ a.vaucelle@w7com.fr

\section{Henri Hudrisier} \\ Université Paris 8, Laboratoire Paragraphe \\ MSH Paris Nord, Leden. \\ 20 avenue du Raincy, \\ F - 94100 Saint Maur \\ henri.hudrisier@wanadoo.fr
}

Titulaire d'un doctorat en Sciences de l'Information et de la Communication de I'Université Paris 8, Alain Vaucelle a rejoint le Département ARTEMIS de Télécom SudParis en 2008 en qualité de professeur associé. Directeur de w7com une société de production plurimédia pendant plusieurs années, il a aussi occupé différentes fonctions pour les chaînes de télévision japonaises. Aujourd'hui ses thématiques de recherche concernent l'interactivité, et plus particulièrement l'interactivité à l'intérieur des médias. Ce champ de recherche couvre les rapports entretenus par les systèmes machiniques et les pratiques d'interactions avec les utilisateurs.

Maître de Conférences HDR, Henri Hudrisier est membre du Laboratoire Paragraphe, Université de Paris 8, du LEDEN (Maison des Sciences de l'Homme Paris Nord), du réseau thématique RES@TICE de l'Agence Universitaire de la Francophonie (AUF). Il est également expert à l'ISO pour la normalisation de l'e-learning (délégué de l'AFNOR en France et de l'AUF), président de L'Alliance Cartago, Vice Président de l'AILF (Association des Informaticiens de Langue Française). Ses axes de recherches : patrimoines multiculturels et multimédias ; normes multimédia ; normes multiécritures ; TEI (Text Encoding Initiatives). 


\title{
Langages structurés et lien social
}

\begin{abstract}
Résumé
Dans cet article les auteurs proposent une approche critique de l'interactivité à travers l'utilisation des langages à balises et des cadres d'application (frameworks). Depuis une vingtaine d'année ces langages réorganisent l'aménagement d'une interactivité pour le développement des technologies de l'information et de la communication. Pour ce faire, normalisateurs, auteurs, utilisateurs s'organisent dans des collèges de gouvernance afin d'aménager l'évolution de ces langages. Cette socialisation devient un des leviers incontournable du développement des systèmes communicants.
\end{abstract}

Mots-clés : interactivité, framework, TEI, SGML, langage à balise

\begin{abstract}
In this article, the authors propose a critical approach to interactivity through the use of markup languages and application frameworks. For twenty years, these languages have been reorganizing how interactivity is laid out in the development of information and communication technologies. Setters of standards, authors, users are organized into colleges of governance in order to design the evolution of these languages. This socialization is one of the essential levers of development of communicating systems.
\end{abstract}

Keywords: interactivity, framework, TEI, SGML, markup language

\section{Resumen}

En este artículo los autores proponen un enfoque crítico de la interactividad a través del uso de balizas y de marcos de aplicación (frameworks). Desde hace veinte años, estos lenguajes habilitan y reorganizan la interacción para el desarrollo de las tecnologías de la información y de la comunicación. Para ello, los creadores, los usuarios y quienes crean estándares, se organizan en organismos de gobernaza con el fin de organizar la evolución de esos lenguajes. La socialización así creada constituye una de los potenciadores esenciales del desarrollo de los sistemas comunicantes.

Palabras claves: interactividad, framework, TEI, balizas, SGML 
Langages structurés et lien social

Les langages informatiques déclaratifs apparus à la fin des années 60 servaient à automatiser et à structurer l'échange de contenus entre systèmes d'informations. Par exemple, Le langage déclaratif SGML (Standard Generalized Markup Language) a été adopté pour structurer et échanger des corpus documentaires volumineux, car il permet de séparer la structure logique du document de sa mise en page. Ces langages dit langages à balises (comme HTML - HyperText Markup Langage, XML - Extensible Markup Language, version simplifiée de SGML, ODA - Office [Open] Document Architecture destiné à remplacer les formats propriétaires de fichiers documents) sont utilisés pour favoriser l'interaction hommes-machines dans des environnements informatiques hétérogènes ${ }^{1}$. Ils sont construits sous la forme de briques modulaires et extensibles (frameworks) basée sur une architecture commune définissant l'étendue de leur application ${ }^{2}$.

II est dès lors possible de gérer les liaisons multiples qui peuvent être établies entre des liens hypertextes, des applications, des machines ou des réseaux.

Par exemple, un fichier vidéo visualisable dans un navigateur pourra donc, au choix de l'auteur, activer des liens hypertextes, du son supplémentaire, un sous-titrage, un lien vers une base de données...

La nature de cette interactivité intrinsèque au langage débouche sur une appropriation de l'interactivité qui n'est pas sans impact sur l'appropriation sociale des machines interactives. Dans ce contexte, qu'entend on par interactivité ?

1 Depuis 1990, date clef du développement des technologies du web, un public très vaste utilise ces langages et leurs dérivés. Le plus célèbre d'entre eux est peut-être HTML, utilisé dans l'édition de page web.

$2 \quad$ Par exemple, le XML a ouvert la voie à des sous-langages fondés sur ce même langage " matrice " répondant à des besoins spécifiques, comme "MathML " (Mathematical Markup Language) pour l'échange des données et des calculs entre mathématiciens. 


\section{La part d'humanité des machines logicielles : entre temps réel et temps différé}

L'interactivité ne découle pas uniquement du «temps réel », c'est-à-dire du caractère immédiat des interactions hommes-machines.

L'interactivité consiste également à définir des préférences sur une interface machine (comme un traitement de texte, un explorateur Internet ou un téléphone portable) et elle renvoie également au contenu informationnel (DTD Document Type Definition ${ }^{3}$, schéma $X M^{4}$ ou framework ${ }^{5}$ ) sans qu'une référence au temps (réel ou différé) ne soit nécessaire.

Enfin, quand nous communiquons avec un logiciel ou une machine, nous sommes également en rapport à la créativité de celui (ou dans le cas le plus fréquent, de ceux) qui les a (ont) mis au point et inventé . $^{\circ}$

Les distinguos commodes et utiles pour établir des typologies de l'interactivité (homme/homme, homme/machine, machine/machine) achoppent ici face à la difficulté que constitue la part d'humanité pensante des machines. Interagir avec une machine, c'est toujours interagir avec de la pensée humaine que l'on parle d'un simple marteau ou d'une application informatique sophistiquée. Dès lors, c'est toujours établir du « lien social » mais d'une autre manière, c'est-à-dire par l'intermédiaire d'une machine.

Avec les technologies industrielles courantes, la situation est proche de la doxa interactive. Un téléphone mobile, un logiciel (comme Word par exemple) nous offrent une palette prédéfinie de possibilités de communication qui guident nos interactions. Dans le cas de la publicité sur internet cette guidance interactivité du rapport entre utilisateurs et concepteurs du site devient encore plus asymétrique. L'utilisateur expose (souvent à son insu) ses besoins, ses interrogations, son profil d'utilisateur susceptible de contrôle (du contrôle parental, au contrôle patronal, voire politique et national).

Dans le cas du téléphone mobile, l'interactivité logicielle est en principe plus simple et moins asymétrique. Nous pouvons souvent redéfinir les

\footnotetext{
$3 \quad$ Une DTD spécifie la structure de données d'un document XML ou SGML.

$4 \quad$ C'est un langage de description de la structure du format XML. Ce schéma permet de vérifier la validité du document XML.

5 En informatique un framework est ensemble ordonnée de briques logicielles en interaction les unes avec les autres permettant de développer une application.

6 Simondon insistait déjà sur l'aspect humain de la machine : toute machine et a fortiori les machines à communiquer sont des réserves d'intelligence, un concentré collaboratif et temporel de pensées et de projets humains qui s'insèrent dans la chaine des activités humaines (Simondon, 1958).
}

tic\&société - 4 (1), 2010 
comportements de l'interface que nous avons organisée au plus près de nos besoins et préférences d'interactions. Cependant, ce système souvent complexe dans son appropriation nous réduit à n'utiliser qu'une infime partie du potentiel interactif des mobiles.

En revanche, une DTD ou un schéma XML exploitent une conception différente de l'interactivité comme une construction qui se présente comme un substrat ou un framework sur lequel l'utilisateur va pouvoir ajouter " entre et dans les balises " ses propres couches d'information, de références, de structures et de sémantiques. Comme on a pu le dire autrefois, les langages structurés sont des shells (des coquilles) dans lesquelles vont pouvoir se nicher des informations propres aux utilisateurs (ou à des groupes d'utilisateurs).

Du fait de leur capacité à passer du mode "lisible par machine » au mode " lisible par l'humain ", les langages structurés offrent des potentiels interactifs très innovants qui ne se développent que lorsqu'ils sont associés avec les contenus spécifiques qui introduisent leurs utilisateurs.

\section{Interactivité et partage collégial}

Chaque communauté d'utilisateurs peut être sous catégorisée en au moins deux collèges hiérarchiques qui correspondent à deux états de ces langages et à deux niveaux d'interactivité.

Le premier concerne les aménageurs du langage et des ressources de contenus. Brillants utilisateurs, ils sont souvent associés à une équipe d'informaticiens spécialisés.

Le second est le collège des utilisateurs courants du langage et des contenus. La frontière n'est jamais totalement hermétique entre ces deux collèges et ces deux niveaux d'interactivité.

Ces collèges se structurent et se différencient selon la compétence à aménager le framework et le réservoir de ressources médiatiques associé. L'enjeu d'aménagement et d'interaction devient un enjeu de gouvernance à la portée des utilisateurs. En effet, tout utilisateur de ces langages peut proposer (très localement, ou à un niveau beaucoup plus global) de devenir l'aménageur soit du framework (et de l'ensemble des balises qui le structurent et qui lui ouvre sa sémantique), soit des ressources liées et instanciées par les balises ${ }^{7}$.

$7 \quad$ Notons que XML a favorisé le développement du logiciel libre et la liberté pour tout utilisateur d'un système informatique interactif d'en devenir aussi un fabriquant en y faisant valoir 
Alain VAUCELLE et Henri HUDRISIER

Le collège le plus englobant (plusieurs centaines de millions de personnes dans le monde) est celui des utilisateurs (directs et surtout indirects) de SGML dont HTML et XML sont des versions dérivées. À des niveaux disparates de compétence, tout utilisateur du web (même occasionnel) rentre dans ce vaste collège.

Viennent ensuite des collèges d'utilisateurs plus spécifiques, comme par exemple:

- les terminologistes et les usagers des terminologies qui utilisent un métamodèle comme le TMF (Terminological Markup Framework, nous y reviendrons);

- les documentalistes ou bibliothèques et leurs usagers qui utilisent un métamodèle comme le Dublin Core (modèle générique utilisé pour la description des ressources numériques) ;

- les créateurs de contenu muséaux qui utilisent le CRM (Conceptual Reference Model) ;

- les créateurs de contenus audiovisuels qui utilisent un métamodèle dont dérivent les normes MPEG ou JPEG ;

- les utilisateurs des TICE qui utilisent un métamodèle comme SCORM (Sharable Content Object Reference Model), mais aussi le LOM (Learning Object Metadata) et bientôt le MLO (Metadata for Learning Opportunities).

Enfin, un méta-collège regroupe un métier, une discipline, des collèges plus spécifiques d'utilisateurs, voire à un niveau encore plus restreint des profils utilisateurs spécialisés. Collèges globaux, utilisateurs et méta-collèges sont articulés les uns aux autres ainsi que les niveaux d'interactivité auxquels ils évoluent. Donnons-en quelques exemples.

Le collège de gouvernance globale : pour une communauté de métier donnée (les terminologistes, les documentalistes, les muséographes, la communauté TEl d'étude des textes, etc.), ce collège utilise XML pour développer un méta-modèle utilisable par telle communauté de métier. Par exemple, le CRM (Common Reference Model), développé sous l'égide de I'ICOM (International Council Of Museums) et du CIDOC (Comité International pour la DOCumentation) est un modèle de référence commun à tous les

ses propres besoins sémantiques et ses propres exigences par ajout de briques logiciels interopérables avec un langage source.

tic\&société - 4 (1), 2010 
musées (art, ethnologie, histoire naturelle, archéologie, sciences et techniques, etc.).

Les collèges de gouvernance thématiques : les collèges d'experts qui développent un modèle commun pour les métiers qu'ils desservent autorisent la formation de sous-communautés plus spécifiques, chacune développant une application particulière. A un niveau encore plus spécialisé, il arrive que des « sous-sous- » collèges soient autorisés à proposer un modèle encore plus spécifique, mais en restant dans le cadre générique du modèle général et en reversant leurs balises spécifiques dans le modèle général.

Mettons qu'un groupe d'utilisateurs lambda aménage un framework. Vont-ils prendre de l'ampleur et vouloir devenir un collège thématique en acceptant les règles de la communauté et de son métalangage ? Resteront-ils au contraire à l'écart, quitte à perdre (partiellement ou totalement) la compatibilité avec le modèle générique ? De cette question dépend l'innovation possible au sein d'un langage structuré, l'interactivité correspondante du langage de même que la socialisation de ses utilisateurs. Le cas le plus exemplaire de ce mode de fonctionnement collégial est celui de la TEI dont l'histoire croise celle du développement d'XML ${ }^{8}$.

\section{La TEI}

La TEl est une application d'organisation collégiale des ressources linguistiques. Elle est gérée et subventionnée principalement par trois associations professionnelles ayant des activités de recherche dans le domaine du traitement de textes par ordinateur, à savoir : I'Association for Computational Linguistics (ACL), l'Association for Literary and Linguistic Computing (ALLC) et l'Association for Computing and the Humanities (ACH). Historiquement l'une des plus anciennes et des plus pérennes, elle permet de paramétrer sur un support SGML (et maintenant XML) des recommandations définissant un en-tête numérique très complet (à la fois commun et spécialisé selon les disciplines et sous-disciplines représentées dans le collège), ainsi que des cadres disciplinaires et sous-disciplinaire de description sémantique des documents. Ces «Recommandations TEI " sont partagées collégialement par les chercheurs et sont régulièrement mises à jour.

8 Des membres de l'équipe de conception de la TEl (dont Lou Burnard) ont participé à la conception et au développement d'XML. 
Alain VAUCELLE et Henri HUDRISIER

\subsection{Historique}

La genèse de la TEI remonte à une conférence tenue au Collège Vassar à Poughkeepsie (Etat de New York) en novembre 1987. Environ trente représentants venant des domaines de l'archivistique, des sciences humaines, de la littérature et de la recherche universitaire ont reconnu l'intérêt et la nécessité d'une normalisation pour l'encodage et l'échange des textes. Leurs discussions ont mené à des recommandations sur la portée, la structure et le contenu que devrait avoir le système d'encodage. La conférence eu pour effet de formaliser une série de principes pour l'élaboration d'un système d'encodage des textes.

Le succès de cette première conférence de Poughkeepsie tient à ce que les participants ont clairement compris que pour parvenir à leurs desseins d'une bibliothéconomie virtuelle associée à des enjeux de recherches, ils devaient s'approprier le substrat numérique, à l'époque le langage SGML. SGML permettait le développement d'un système simple, flexible, hautement interactif et ayant la capacité de satisfaire tous les besoins de mise en réseau des ressources documentaires (bibliothèque numérique) et de traitements de texte standardisés adaptés à la grande diversité des usages. SGML devaient également permettre de remplir les besoins qui avaient déjà été identifiés, à savoir, la capacité d'exprimer des distinctions ou des chevauchements hiérarchiques complexes, assurer l'indépendance des données vis-à-vis des différents logiciels et des plateformes informatiques et la compatibilité avec les normes existantes.

Cette conférence débouche sur la Text Encoding Initiative (juin 1988) soutenue financièrement par le National Endowment for the Humanities. Quatre comités sont mis sur pied par des chercheurs d'Europe et d'Amérique du Nord pour étudier les questions relatives à la documentation des textes électroniques, à leur représentation, à leur analyse, leur interprétation et à la syntaxe du métalangage. Ce travail a mené à l'élaboration d'une DTD et des Guidelines for Electronic Text Encoding and Interchange. 
Langages structurés et lien social

\subsection{La TEI Lite}

La DTD TEI Lite est une sélection des éléments, attributs et paramètres de la DTD complète issue de la TEI. Elle définit les éléments minimums que chaque utilisateur de la TEI devrait connaître ${ }^{9}$.

On l'a souligné pour les téléphones mobiles - un potentiel d'interactivité maximal peut compliquer, voire paralyser la capacité d'interaction de certains utilisateurs. De ce fait, la TEI est souvent utilisée ad minima soit sous forme " allégée » (Lite), soit limitée à son seul « en-tête » (header).

\subsection{L'en-tête de la TEI (TEI header)}

La TEI limité à son seul « header » est un format particulièrement adapté à la création de bibliothèques numériques virtuelles.

L'en-tête de la TEI décrit un document balisé permettant aux utilisateurs d'avoir de l'information sur la ou les source(s) d'un texte, les principes utilisés pour le balisage, l'historique des révisions et modifications apportées au texte. Ces informations sont nécessaires autant pour les chercheurs qui utilisent les textes que pour les catalogueurs. Aucun document n'est conforme à la TEI s'il ne comporte pas la balise <teiHeader $>$.

$\mathrm{Ce}<$ teiHeader> permet de distinguer le document physique source (un livre, un périodique, une bande vidéo, une transcription d'entretien) de sa transformation en un nouveau document numérique. Par exemple, si une bibliothèque numérique commentait la présente contribution, le document d'origine deviendrait lui même un nouveau document (une glose) qui aurait son " header » numérique distinct, mais qui garderait « la trace » du document de référence 10 .

La TEI (complète ou allégée) et son " header » définissent premier niveau d'interactivité. L'interaction des chercheurs qui collaborent pour paramétrer et modéliser ces langages sur la base d'outils spécialisés (par exemple : TEI

$9 \quad$ Plusieurs applications utilisent la TEI Lite. Les Oxford Text Archive l'utilisent pour convertir vers XML les textes déjà numérisés dans un autre format tout comme les Electronic Text Centers de I'Université de Virginie et de I'Université du Michigan I'utilisent pour le balisage de tous leurs documents. La Text Encoding Initiative utilise elle-même TEI Lite pour sa documentation technique. En France on peut citer Grisemine, remplacé aujourd'hui par IRIS (Université de Lille sur la Littérature grise) et le projet concernant le dépôt national des thèses.

10 http://www.tei-c.org/Guidelines/P4/html/HD.html 
Alain VAUCELLE et Henri HUDRISIER

Pizza11) constitue un second niveau d'interactivité. Ces chercheurs opèrent selon des "lignes directrices " plutôt que des règles immuables afin de garantir l'adaptation optimale des langages aux types de textes ${ }^{12}$. Les textes sont analysés de façon collaborative sur la base de schémas ou DTD spécialement mises en oeuvre pour tel type de texte (par exemple TEldrama pour la prose et le théâtre, TEltranscribed speech pour les ressources orales).

A l'exemple de la TEI, l'enjeu majeur de l'interactivité des langages structurés est de favoriser les interactions collégiales à géométrie variable permettant la maitrise collective d'objets complexes et soumis à l'évolution permanente de ces langages.

\section{Enjeux normatifs, sémantiques et régulatifs}

La force des langages structurés, leur potentiel interactif tient donc à l'intelligence collective qu'ils peuvent mobiliser, c'est-à-dire un lien de collaborativité entre chercheurs (Ide, Véronis, 1996) susceptible de favoriser l'automatisation de la génération d'informations interactives. Les premiers langages structurés et la TEI aura initié ce mouvement, dont les développeurs seront soutenus par des bibliothèques et de nombreux éditeurs (notamment ceux qui éditent des annuaires, des dictionnaires, des catalogues de vente).

L'ouverture vers le grand public sera favorisée par la mise en place du Web. En construisant une application SGML d'abord réservée aux physiciens, Tim Berners-Lee du CERN paramètre un ensemble d'informations scientifiques réactualisables et collectivement constructibles. De plus, il imagine lier ces informations aux messageries Internet déjà très populaires depuis une dizaine d'années chez les scientifiques, mais qui jusqu'alors n'étaient que très peu interactives. II définit une DTD de SGML (HTML) et donne le coup d'envoi de l'interactivité telle que nous la connaissons aujourd'hui.

La communauté qui construira XML offre encore plus de possibilités pour le grand public de déployer une interactivité sémantique restée limitée avec

\footnotetext{
11 http://www.tei-c.org/pizza.html

12 Ces lignes directrices se présentent sous forme de conventions pour la description physique et logique de plusieurs genres de textes, comme par exemple la gestion des références croisées à l'intérieur et à l'extérieur d'un document, l'identification arbitraire de segments de textes, l'alignement d'éléments parallèles occupant la même place dans la structure hiérarchique du texte, le chevauchement des hiérarchies, la gestion des liens hypertextes vers des données textuelles, sonores ou visuelles (norme HyTime - Hypermedia/Time-based Structuring Language).
}

tic\&société - 4 (1), 2010 
Langages structurés et lien social

HTML13. Aujourd'hui, le TMF (Romary, 2001), SCORM (Pernin, 2004), le Dublin Core (Ide, Cherhal, 2004) esquisse le futur de cette interactivité des langages structurés, où il ne s'agit plus seulement de faire collaborer les hommes et les machines à l'amélioration de leurs interactions, mais où il s'agit également d'augmenter la capacité des machines à interpréter l'information échangée. C'est un pas non négligeable vers l'intégration des différents logiciels, des dispositifs machiniques, des diverses modalités sensorielles et médiatiques (images, sons, graphiques, réalité virtuelle, écriture, oralité, monétique...) dans un même univers d'interactivité, qui doit garantir parallèlement le maintien de la diversité des langages structurés et des (sous-)collèges associés.

Comme le fait remarquer Saussure à propos des langues naturelles, l'immutabilité d'une langue dépend paradoxalement de sa mutabilité. En effet, pour que les locuteurs se comprennent, le code doit être arbitraire et intangible, immuable. Cependant, les langues doivent évoluer pour se maintenir, elles doivent pouvoir muter. II en va de même pour les langues artificielles : leur mutabilité est aussi indispensable que leur immuabilité. Comme les langues naturelles, les langues artificielles doivent évoluer en permanence. Les efforts de la normalisation sont là pour réaménager périodiquement l'ensemble du système communicationnel mondial, lui permettre de fonctionner malgré sa profusion d'innovations et malgré les standards exclusifs qui protègent les recherches et développements innovants relatifs à des demandes adaptatives très souvent disparates. On peut ainsi poser comme hypothèse que la normalisation mondiale des TIC est très similaire mais aussi très complémentaire de la préservation de la pluralité linguistique des systèmes d'information. Cette pluralité inhérente à la société-monde qui conditionne la capacité d'innover de notre société détermine également le devenir de cette interactivité innovante des langages structurés.

\section{Conclusion}

Les strates successives de typologies et de caractérisation savante des médias constituent une première piste d'investigation. McLuhan distinguait les médias froids des médias chauds (McLuhan, 1977). Quid de cette typologie à l'ère de la convergence globale du multimédia et du numérique ? A sa suite un auteur comme Cloutier (Cloutier, 1973) faisait date en opposant les self-médias (photographie, ordinateur, téléphone) aux mass médias. II n'y a pas si

13 Notons que cette ouverture sémantique de $\mathrm{XML}$ a sans doute été ouverte trop librement pour un grand public ignorant forcément les enjeux de l'arbitraire du code, de son partage, de son immuabilité et de sa mutabilité. 
longtemps le concept de "prothèse médiatique " caractérisait les médias interactifs comme d'ailleurs le téléphone, l'automobile ou les outils de la réalité virtuelle qui augmentent non seulement le potentiel des capacités physiques de l'être humain, mais aussi ses capacités communicationnelles. En créant le Web, Tim Berners-Lee avait propulsé le SGML (devenu HTML) à un niveau d'appropriation sociale tel que ce langage réputé technique et spécialisé devenait un des leviers incontournable de la mondialisation de la communication.

La numérisation intégrale de toute la communication et de l'information dans le sillage de la numérisation programmée (Vaucelle et al., 2008) de la TV et la radio nous semble constituer une mutation en devenir de l'interactivité au moins aussi importante que celle du Web. Pour nombre de spécialistes de l'observation des médias, le véritable "Web sémantique futur " ne saurait être confondu avec les perfectionnements du Web actuel imaginés par des chercheurs en informatique. II s'appuiera certainement sur l'appropriation sociale par des individus aujourd'hui enfants ou adolescents qui manipuleront des objets médiatiques interactifs mutants mais éminemment convergents. Le langage substrat sur lequel se développent les médias numériques de demain est également dérivé d'XML. II fait partie des normes de la famille MPEG (MPEG-4, 7 et 21 ; Vaucelle, 2009). La capacité d'interaction de ces « normes langages » est telle qu'elle permet d'intégrer des niveaux d'interactions aujourd'hui très hétérogènes : la téléphonie, la e-mobilité, le e-juridique, le ecommerce, la réception télévisuelle et radio interactive, la simulation 3D etc.

Comme on le voit, le devenir de l'interactivité s'inscrit au coeur de multiples paradoxes, où elle dépend à la fois des développements technologiques industriels concertés mais concurrents et des mutations langagières sociétales attendues mais par nature relativement imprévisibles.

\section{Références bibliographiques}

CHERHAL É., 2004, "Présentation des standards : le Dublin Core (DC) », Communication rédigée par l'auteur suite à sa communication orale le 16 novembre 2004, ENSSIB, <http://www.enssib.fr/bibliothequenumerique/document-1236>, dernière consultation le 25 octobre 2009.

CLOUTIER J., 1973, L'ère d'Emerec. La communication audio-scripto-visuelle à l'heure des self-média, Canada, Presses de l'Université de Montréal.

IDE, N., J. VÉRONIS, 1996, «Présentation de la TEI : Text Encoding Initiative », Cahiers GUTenberg, vol. 24, p.4-10, 
<http://www.gutenberg.eu.org/pub/GUTenberg/publicationsPDF/24presentation.pdf>, dernière consultation le 25 octobre 2009.

MARTINET A., 1964, Éléments de linguistique générale, Paris, Armand Colin.

MCLUHAN M., 1977, La galaxie Gutenberg - La genèse de l'homme typographique, Paris, Gallimard.

PERNIN J., 2004, « LOM, SCORM et IMS-Learning Design : ressources, activités et scénarios ? ", Compte-rendu rédigé par l'ENSSIB à partir d'une transcription de la communication orale de Jean-Philippe Pernin le 16 novembre 2004, ENSSIB, <http://www.enssib.fr/bibliothequenumerique/document-1239>, dernière consultation le 25 octobre 2009.

ROMARY L., « Un modèle abstrait pour la représentation de terminologies multilingues informatisées TMF Terminological Markup Framework ", Cahiers GUTenberg, $\mathrm{n}^{\circ} 39-40$, Mai 2001, p. 81-88, <http://hal.archivesouvertes.fr/docs/00/10/05/88/PDF/Romary.pdf>, dernière consultation le 25 octobre 2009.

SIMONDON G., 1958, Du mode d'existence des objets techniques, Paris, Aubier.

VAUCELLE A., F. PRETEUX, M. BEN HENDA, H. HUDRISIER, 2008, « Les normes des TICE du XXlème siècle », à paraître dans Actes du colloques CIUEN 2008, l'Université à l'ère du numérique, du 10 au 12 décembre, Bordeaux.

VAUCELLE A., 2009, « MPEG-21 : la norme des TICE du XXlème siècle ? ", dans Actes du colloques H2PTM'09 Rétrospective et perspective : 1989 2009, du 30 septembre au 2 octobre, Saint-Denis (93), Hermès, p.115-121. 